\title{
Theoretical and experimental investigation of shock wave stressing of metal powders by an explosion
}

\author{
A.E. Buzyurkin ${ }^{1, a}$, E.I. Kraus ${ }^{1}$, and Ya.L. Lukyanov ${ }^{2}$ \\ 1 Khristianovich Institute of Theoretical and Applied Mechanics of Siberian Branch of RAS, \\ Instituskaya str., 4/1, 630090 Novosibirsk, Russia \\ 2 Lavrentiev Institute of Hydrodynamics of Siberian Branch of RAS, Lavrentev pr., 15, \\ 630090 Novosibirsk, Russia
}

\begin{abstract}
Joint theoretical and experimental investigations have allowed to realize an approach with use of mathematical and physical modeling of processes of a shock wave loading of powder materials. Hugoniot adiabats of the investigated powder have been measured with a noncontact electromagnetic method. The mathematical model of elasticplastic deformation of the powder media used in the investigation has been validated. Numerical simulation of shock wave propagation and experimental assembly deformation has been performed.
\end{abstract}

\section{Introduction}

Methods of explosive loading of powder materials in conservation ampoules are applied in order to obtain new materials including composite ones with the unique physical and mechanical properties. In addition, these methods can be used to study phase transitions occurring in materials at high pressures and temperatures taking place behind shock waves, as well as for the synthesis of metastable phases.

In recent decades, significant development has been achieved in such a scientific and technical branch of materials science as powder metallurgy. This term is currently understood a whole complex of problems connected with the design of materials and products from metal and nonmetal powders. Interest in these problems is quite understandable since the opportunity to create new classes of materials with unique and controllable properties which can not be obtained by ordinary metallurgy methods has arisen.

A special place in the powder metallurgy is occupied by explosive compaction of powder materials. It is easy to explain the strong interest in the explosive compaction. It consists in the fact that virtually all the methods of composite materials' production from powder mixtures lead to a change in initial material properties due to high temperatures and relatively long duration of the process.

Since the powders being in the form of granules, fibers, needles and ribbons, possessing the necessary properties in the initial state, can not be used directly to produce semi-finished products or components, the methods of compaction of these materials perform two tasks at once. On the one hand the compaction changes the shape and size of the powders, and on the other hand it produces the material itself. From this point of view, the short exposure to high temperatures and pressures during explosive compaction allows, in general, to keep the original structure and properties of the components. At the same time, varying of the intensity and time exposure to high pressure and temperature in shock compression allows to modify, if necessary, the structure and properties of the compacts a controlled manner.

\footnotetext{
a e-mail: buzjura@itam.nsc.ru

This is an Open Access article distributed under the terms of the Creative Commons Attribution-Noncommercial License 3.0, which permits unrestricted use, distribution, and reproduction in any noncommercial medium, provided the original work is properly cited.
} 


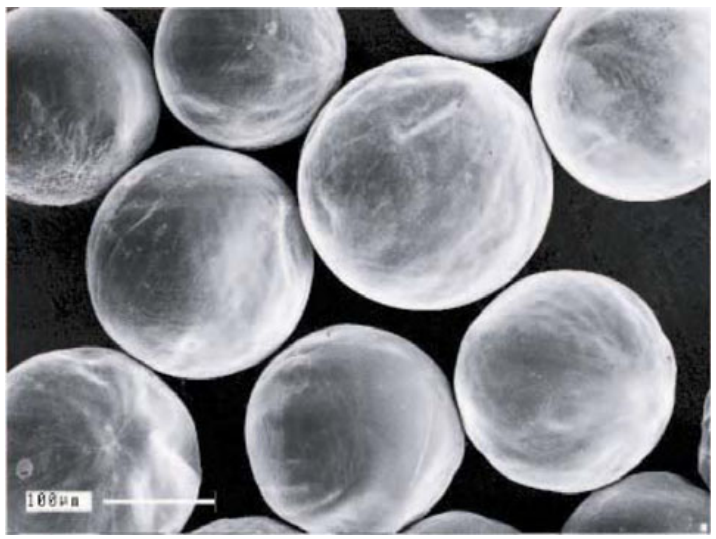

Fig. 1. Initial cooper powder.

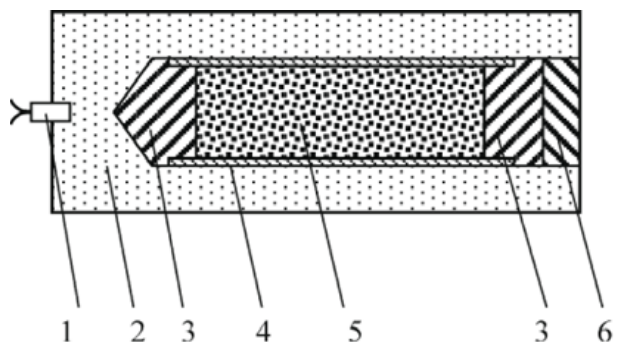

Fig. 2. Scheme of the explosive compaction: 1 - detonator, 2 - a charge of explosive, 3 - stoppers, 4 - container, 5 - powder, 6 - spall element.

The loading of the powder materials in the conservation ampoules can be carried out by means of both plane and oblique shock waves. Each of the methods has its pros and cons. The explosive loading by oblique shock wave is characterized by high values of shear strain, in comparison with the plane impact, which leads to stronger bonds between the compacted particles. In addition, this scheme allows to obtain the compacts not only in the form of plates, but pipes, rods, cones, etc as well. One can also get the compacts of large sizes. The loading by plane shock waves allow to vary the pressure and temperature behind the shock front in a wider range and to reach much higher values of these parameters. At the same time, the method is more material-consuming and has limitations on the size of the loaded samples.

\section{Experimental study of the compact structure}

Experiments on the explosive compaction have been conducted by a cylindrical scheme without a central rod. The powder has consisted of particles of nearly spherical shape and with size of 145$310 \mu \mathrm{m}$ (Fig. 1). Bulk density of the powder has been in all experiments for copper equal to $-5.0 \pm$ $0.05 \mathrm{~g} / \mathrm{cm}^{3}$, for aluminum $-1.4 \mathrm{~g} / \mathrm{cm}^{3}$, tungsten $-10.5 \mathrm{~g} / \mathrm{cm}^{3}$.

The explosive compaction has taken place under the action of detonation products of explosive contact charge. The charges have been made of ammonite, RDX and mixtures of ammonite with RDX and ammonium nitrate in different proportions. The explosive velocity has been measured by an electrocontact method and has varied in the range from 2.2 to $6.2 \mathrm{~km} / \mathrm{sec}$. In the all experiments the condition $\delta \geq r$ has been kept, where $\delta$ is the layer thickness, $r$ - the outer radius of the container with the powder. The wall of the container has been thin compared with the thickness of the explosive layer and diameter of the powder sample. The compact structures have been studied using an optical microscope NEOPHOT and scanning electron microscope LEO-420. The experimental scheme is shown in Fig. 2. 
Preliminary the non-contact electromagnetic method [1] has been used to measure the Hugoniots of the investigated powder. This technique allows in one experiment to record both the velocity of the shock wave and mass velocity behind its front.

Hugoniots adiabats of the investigated powders are shown in Fig. 3 as the dependence of the shock velocity on the mass velocity behind the shock front.

The structures of the compact cross sections and its diffractograms have been studied using optical and scanning electron microscopes. In Fig. 4 the structure of the cross sections of the compacts near the axis of the ampoule for different values of the detonation velocity of the charge is presented. It is evident that with the charge detonation velocity increasing the structure of the central zone changes qualitatively.

Figure 5 shows the difractograms of the compacts obtained by the detonation velocity of 3.95 and $5.26 \mathrm{~km} / \mathrm{sec}$. In the first case, the deformation of the particles takes place in a quasistatic regime, and in the second - in a dynamic one, which is characterized by the presence of material jets and a stronger bond between the particles.

It can be noticed that by Mach wave approach to the steady state, the velocity of it is equal exactly to the detonation velocity of the explosive charge. Using the shock relations and the equation of state, such as Mie-Gruneisen for instance, the critical shock speed $D_{\text {melt }}$ can be found, at which the powder material behind the plane shock wave is heated up to the melting point. Then for an axisymmetric explosive compaction scheme, selecting a charge of the explosives with a detonation velocity less than $D_{\text {melt }}$, a homogeneous compact can be obtained.

Thus, a simple criterion for producing of the homogeneous compact in irregular regime of the shock reflections in the axisymmetric case may be offered. Note that this criterion is applicable for powders with high enough bulk density, equal to about half the density of a monolithic material or higher. At high initial porosity $D_{\text {melt }}$ can be so small that during the compaction with the aid of the axisymmetric scheme with the same detonation velocity, the material at the periphery of the container may not be compressed to the density of monolithic material. Therefore, for the compaction without prior premolding powders with a particle shape close to spherical are preferred, as they have high bulk density.

\section{Numerical simulation of the explosive loading}

For numerical simulation of the shock waves propagations the full system of equations of porous elastic-plastic material deformation is solved [2].

The problem statement is shown in Fig. 6.

The influence of the explosion products on the powder is simulated by pressure applied to the upper boundary of the container. The magnitude of the applied pressure is determined by the formula that approximates the behavior of the pressure by the expansion of the detonation products [3]:

$$
P(t)=P_{H} \exp \left(-(t-x / D) / t_{1}\right), \quad t_{1}=\sqrt{\frac{3\left(\gamma_{e}+1\right)}{4\left(\gamma_{e}-1\right)}} \frac{\delta_{e}}{D},
$$

here $\delta_{e}$ is the thickness of the explosive layer, $\gamma_{e}-$ the adiabatic exponent of the detonation products. Since the problem is symmetric, a half of the experimental assembly is considered. The symmetry axis is the axis of the container with the powder. On the symmetry axis rigid wall boundary conditions are set. The right boundary is considered to be free of stress, and at the left boundary condition of a rigid wall is put. Computation of the contact boundaries is performed by using a symmetric algorithm [4]. The calculations are carried out by the M. Wilkins scheme [5]. The shock wave propagates from left to right. Geometric dimensions and values of the physical parameters correspond to the experimental data mentioned above.

In this paper a few-parametric equation of state is applied [6], which has allowed to simulate shock-wave processes with a minimal number of physical parameters as the initial data.

$$
E=E_{x}+c_{v, l} T+\frac{1}{2} c_{v, e 0} T^{2}\left(\frac{V}{V_{0}}\right)^{2 / 3}
$$


EPJ Web of Conferences
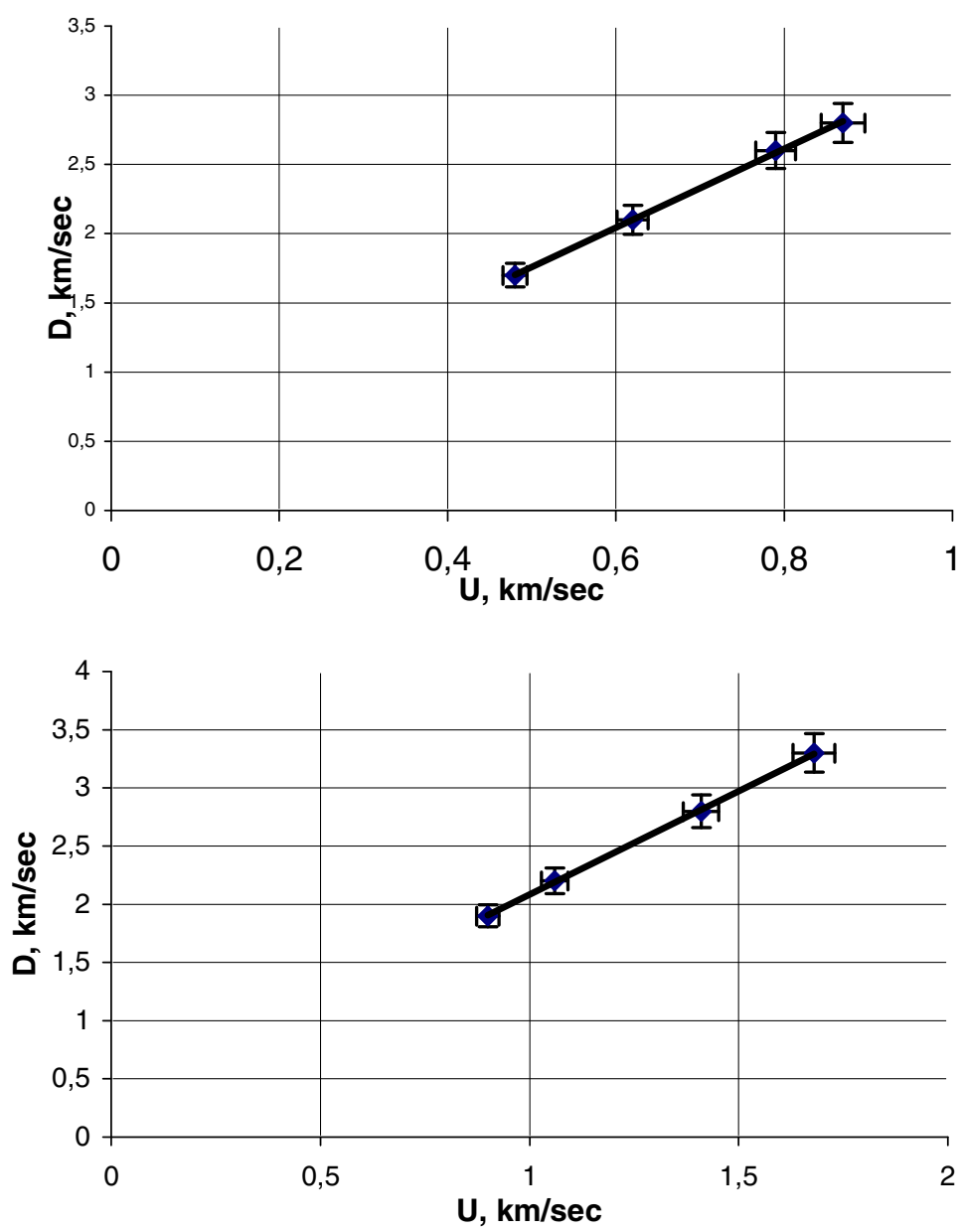

a)

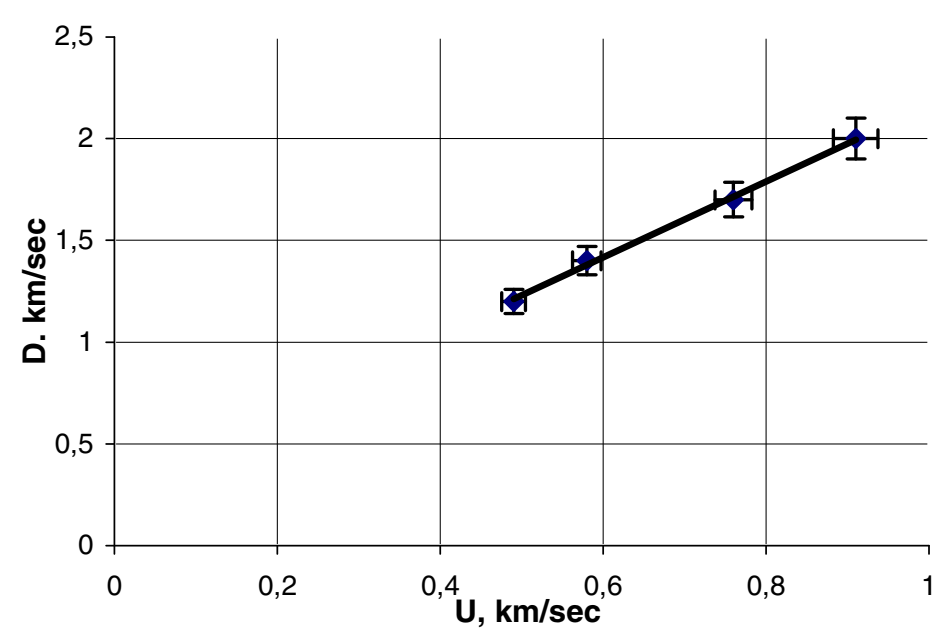

b)

c)

Fig. 3. Hugoniot adiabats of the investigated powders. D - shock wave velocity, U - mass velocity. a) copper with an initial density of $6.2 \mathrm{~g} / \mathrm{cm}^{3}, \mathrm{~b}$ ) aluminum with an initial density of $1.4 \mathrm{~g} / \mathrm{cm}^{3}$, c) tungsten with an initial density of $10.5 \mathrm{~g} / \mathrm{cm}^{3}$. 
New Models and Hydrocodes for Shock Wave Processes in Condensed Matter

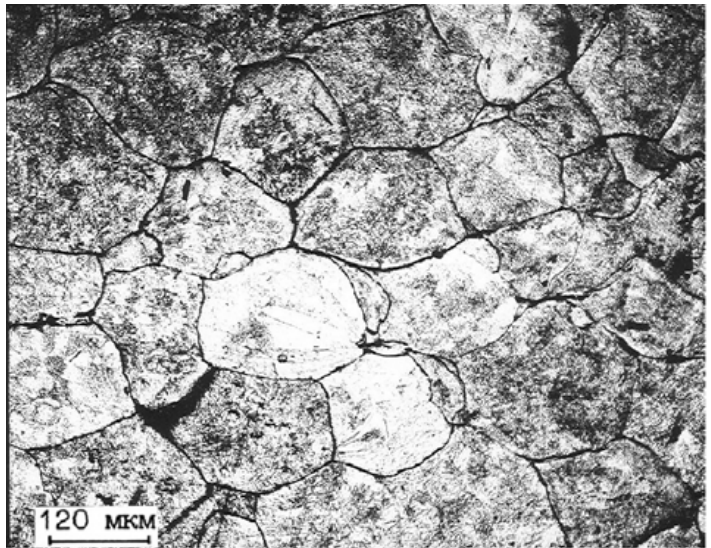

a)

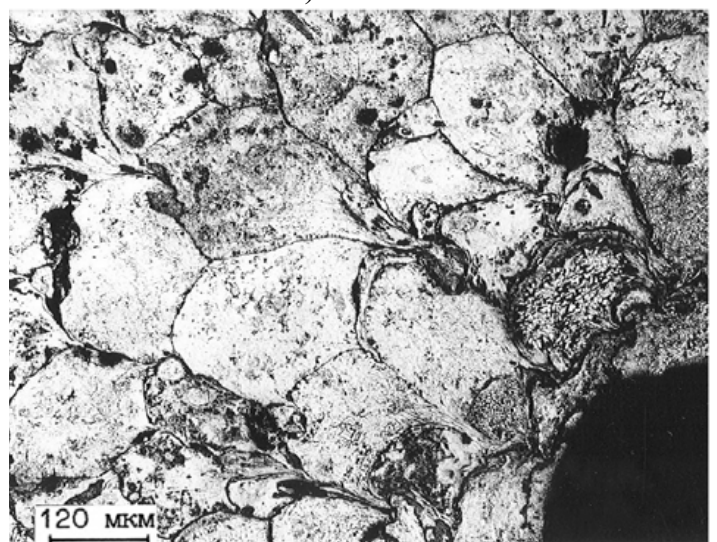

c)

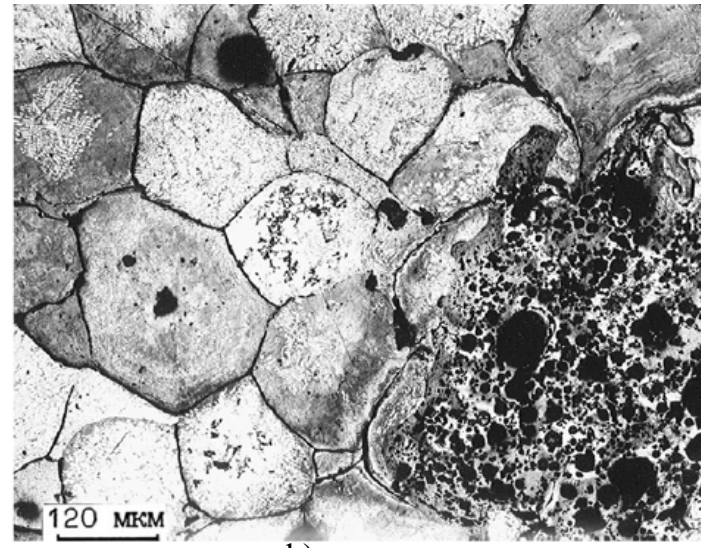

b)

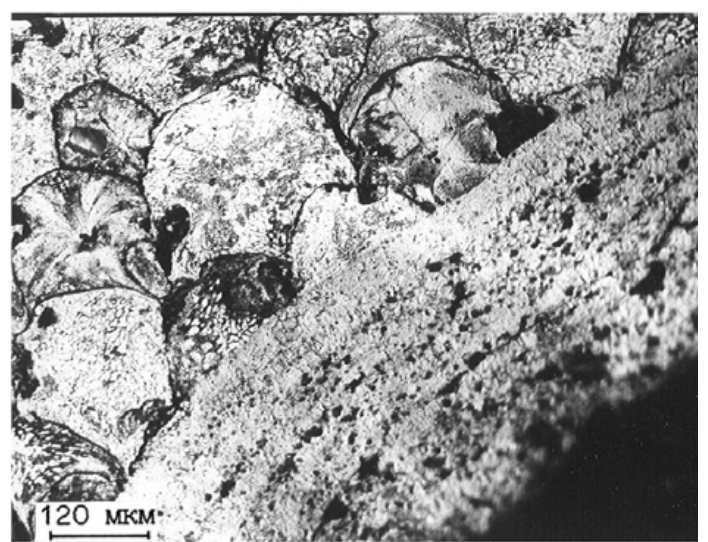

d)

Fig. 4. Structures of the cross-sections of the compacts in vicinity of the ampoule axis for a) $D=2.75 \mathrm{~km} / \mathrm{sec}$, b) $D=3.95 \mathrm{~km} / \mathrm{sec}$, c) $D=5.26 \mathrm{~km} / \mathrm{sec}$, d) $D=5.84 \mathrm{~km} / \mathrm{sec}$, here $D$ - the detonation velocity of an explosive charge.

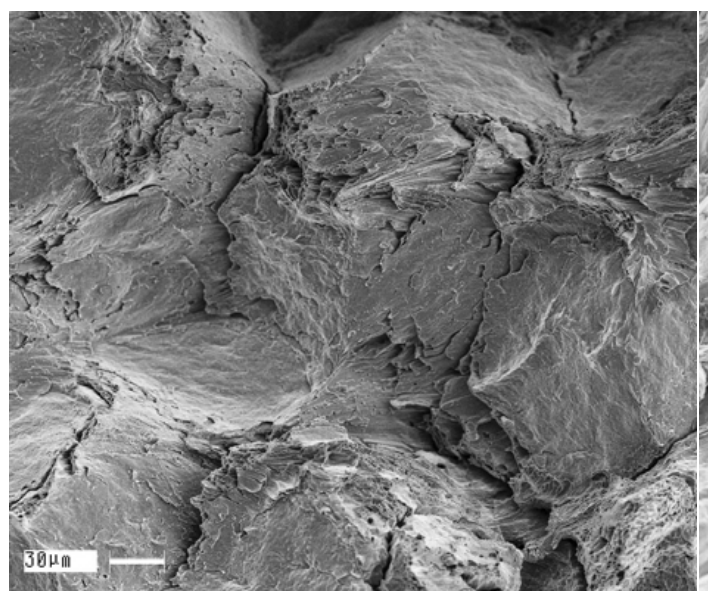

a)

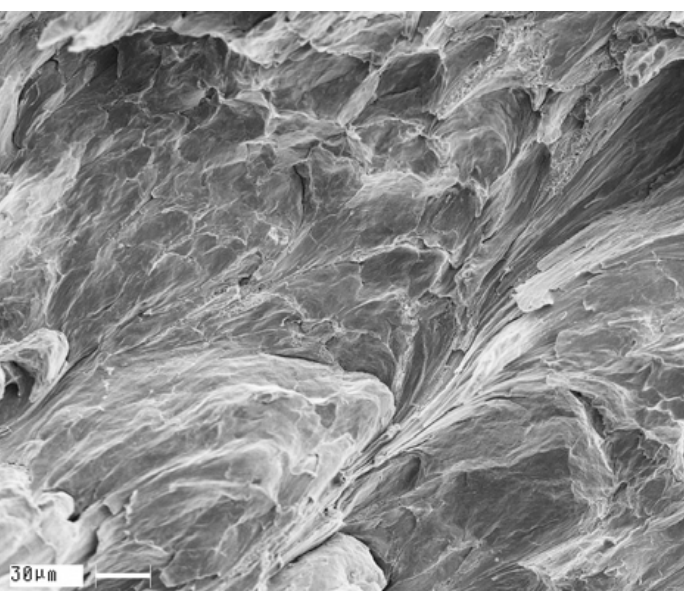

b)

Fig. 5. Difractograms of the compacts at far from axis for: a) $D=3.95 \mathrm{~km} / \mathrm{sec}$, a) $D=5.26 \mathrm{~km} / \mathrm{sec}$. 


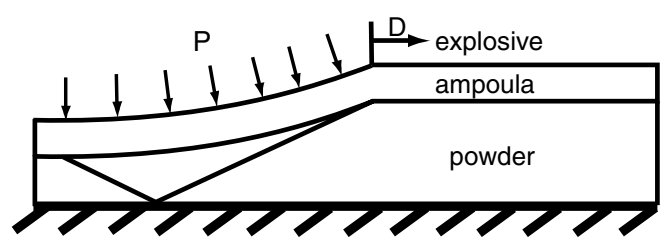

Fig. 6. The computational scheme.

$$
P=-\left(\frac{\mathrm{d} E_{x}}{\mathrm{~d} V}\right)+\frac{\gamma_{l} c_{v, l} T}{V}+\frac{1}{3} \frac{c_{v, e 0} T^{2}}{V}\left(\frac{V}{V_{0}}\right)^{2 / 3}
$$

or, in terms of free energy

$$
F(V, T)=E_{x}(V)+c_{v, l} T \ln \left(\frac{\theta(V)}{T}\right)-\frac{1}{2} c_{v, e 0} T^{2}\left(\frac{V}{V_{0}}\right)^{2 / 3},
$$

where $P_{x}$ and $E_{x}$ - pressure and specific internal energy of the zero isotherm, $T$ - temperature, $c_{v}=$ $c_{v, l}+c_{v, e}-$ heat capacity at constant volume, $\theta(V)-$ the Debye temperature.

The equation of state presented here is based on the dependence of the Gruneisen coefficient $\gamma$ on the volume [7]

$$
\begin{gathered}
\gamma(V)=2 / 3-2 /\left(1-a V_{0} / V\right), \\
a=1+2 /\left(\gamma_{s}-2 \beta\right)+2 P_{t, 0} / K_{s}
\end{gathered}
$$

where $\gamma_{s}=\beta K_{s} V_{0} / c_{v}, K_{s}$ - adiabatic bulk modulus, $\beta$ - coefficient of thermal expansion, $P_{t, 0}-$ heat pressure under the normal conditions.

To find the elastic curves a generalized model describing the Gruneisen coefficient $\gamma(V)$ is used:

$$
\gamma(V)=-\left(\frac{2-t}{3}\right)-\frac{V}{2}\left[\frac{\mathrm{d}^{2}\left(P_{x} V^{2 t / 3}\right) / \mathrm{d} V^{2}}{\mathrm{~d}\left(P_{x} V^{2 t / 3}\right) / \mathrm{d} V}\right]
$$

at $\mathrm{t}=0$ the equation corresponds to the Landau and Slater theory $[7,8]$, at $\mathrm{t}=1$ it corresponds to the Dugleyla and MacDonald hypothesis [9], and at $t=2$ to the theory of free volume [10].

In the physics of shock waves a method of calculating the pressure at the Hugoniot adiabat of the porous material by pressure on the "reference" Hugoniot adiabat of monolithic material [11] is known:

$$
P_{h, p}=\frac{P_{h}\left(1+0.5 \gamma(V)\left(1-V_{0} / V\right)\right)}{1+0.5 \gamma(V)\left(1-V_{00} / V\right)}
$$

Here $V$ is the specific volume of the Hugoniot adiabats, $V_{0}$ and $V_{00}$ are specific volumes of monolithic and porous materials, respectively, at the normal initial conditions.

With the results obtained computed Hugoniot adiabats for copper and aluminum powders have been constructed at different values of initial porosity (Fig. 7). The presented comparison of the results obtained in this study with known experimental data [12-15] shows that the error in calculating of the parameters of the adiabat does not exceed $5-10 \%$.

Figure 8 shows the temperature distribution over the sample thickness through the compacting zone for several values of the detonation velocity. It can be clearly seen that at the higher speeds there is considerable heterogeneity in the temperature distribution over the thickness of the sample. In vicinity of the sample axis the temperature has a higher value than in distance. With the loading rate decreasing, uniform distribution of the parameters over the sample thickness is observed. As a result, it turns out to be possible to obtain homogeneous compacts by the cylindrical loading scheme. 
New Models and Hydrocodes for Shock Wave Processes in Condensed Matter

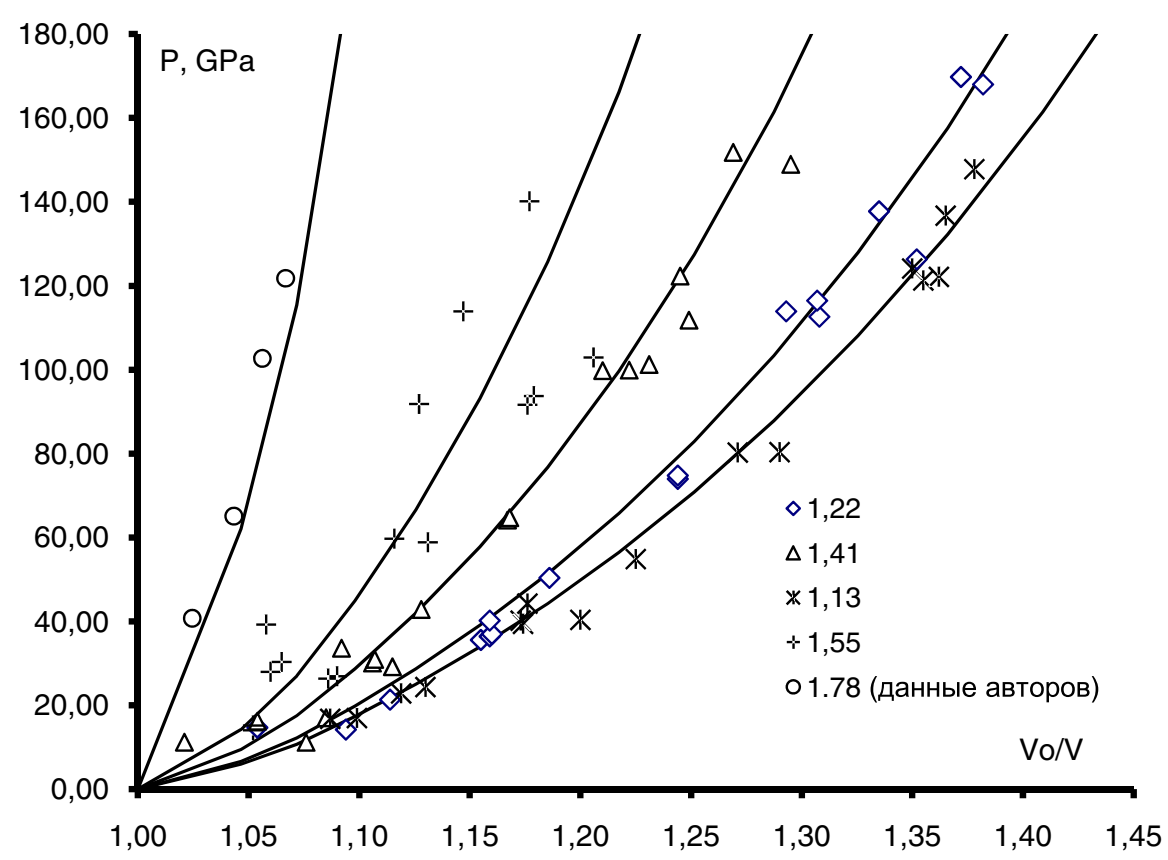

a)

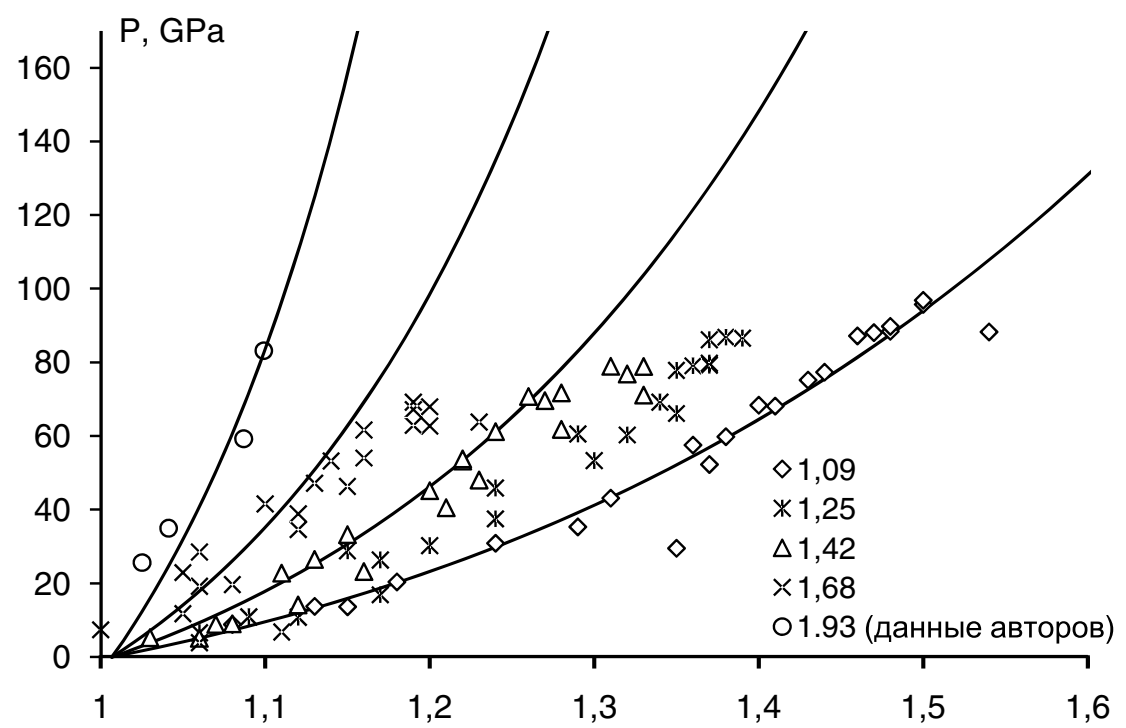

b)

Fig. 7. Hugoniot adiabat of porous material for different values of initial porosity. Calculated and experimental data. a) copper and b) aluminum.

\section{Conclusions}

Joint theoretical and experimental studies have allowed to implement an approach that uses mathematical and physical simulation of shock-wave loading of powdered materials. The Hugoniot adiabats of the investigated powder has been measured by non-contact electromagnetic method. The mathematical model of elastoplastic deformation of powder medium has been validated. A numerical simulation of 


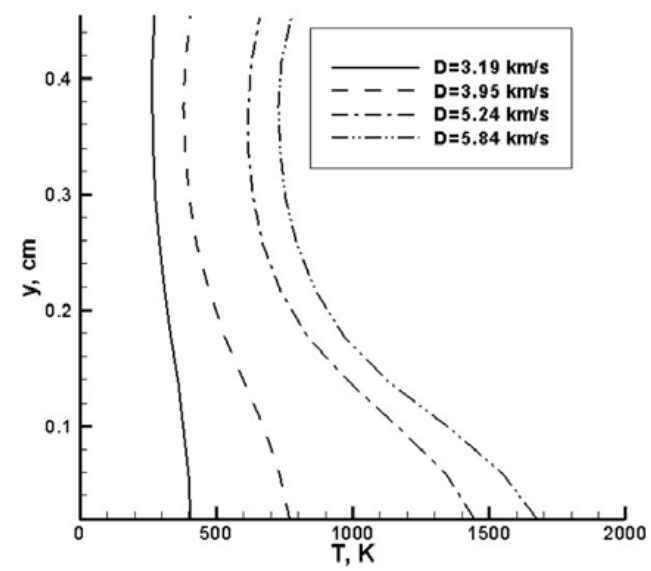

Fig. 8. The temperature distribution over the sample thickness in the compacted zone for different values of detonation velocity. The material is copper.

shock wave propagation and deformation of the experimental assembly has been performed. The temperature distributions over the sample thickness in the compacted zone for several values of detonation velocity show that at higher speeds there is considerable heterogeneity in the temperature distribution over the sample thickness. Near the axis of the sample the temperature has a higher value than in distance from it. With the loading rate decrease uniform distribution of the parameters over the sample is observed. Thus the loading rate is defined at which it becomes possible to obtain homogeneous compacts in their properties by the cylindrical loading scheme.

The work has been financially supported by Ministry of Education and Sciences of Russian Federation, Federal Target Programme "Scientific and educational personnel of innovative Russia", contract \#P356 from 30.07.2009

\section{References}

1. Yakovlev I.V., Pai V.V., Zlobin S.B., Lukyanov Ya.L., Kuzmin G.E., Physical Mesomechanics. 4, 4 (2001)

2. Kiselev S.P., Fomin V.M., PMTF. 6 (1993) (in Russian)

3. Yakovlev I.V., Pai V.V., Kuzmin G.E., FGV, 31, 3 (1993) (in Russian)

4. Fomin V.M. et al., Vysokoskorostnoe vzaimpdeistvie tel. (Novosibirsk. Izdatelstvo SO RAN, 1999) (in Russian)

5. Mark L. Wilkins Computer Simulation Of Dynamic Phenomena (Springer-verlag 1999)

6. Kraus E.I,. Vestnik NGU, 2, 2 (2007) (in Russian)

7. Slater I.C. Introduction in the chemical physics (New-York-London: McGraw Book company, Inc., 1935)

8. Landau L.D., stanyukovitch K.P., DAN USSR, 46, (1945) (in Russian)

9. Dugdale J.S., McDonald D., Phys. Rev., 89 (1953)

10. Zubarev V.N., Vaschenko V.Ya., FTT, 5, (1963) (in Russian)

11. Ray Kinslow (Ed.) High Velocity Impact Phenomena (Academic Press, 1970)

12. van Thiel M. (Ed.), Compendium of shock wave data, (Livermore: Lawrence Livermore Laboratory Report UCRL-50108, 1977)

13. Trunin R.F., Simakov G.V., Sutulov Yu.N. et al., ZhETF, 96 (1989) (in Russian)

14. Marsh S.P. (Ed.) LASL Shock Hugoniot Data (Berkeley etc.: Univ. California Press, 1979)

15. Kormer S.B., Funtikov A.I., Urlin V.D.,Kolesnikova A.N., ZhETF, 42 (1962) (in Russian) 